Running title: Bilingualism and cognitive decline

\title{
Bilingualism and cognitive decline: A story of pride and prejudice
}

Evy Woumans ${ }^{1}$, Jan Versijpt ${ }^{2}$, Anne Sieben ${ }^{3}$, Patrick Santens $^{3}$, \& Wouter Duyck ${ }^{1}$

${ }^{1}$ Department of Experimental Psychology, Ghent University, Gent, Belgium.

${ }^{2}$ Department of Neurology, Brussels University Hospital, Vrije Universiteit Brussel, Brussels, Belgium

${ }^{3}$ Department of Neurology, Ghent University Hospital, Ghent University, Ghent, Belgium

\section{Acknowledgement}

The work of Evy Woumans is supported by the Department of Experimental Psychology at Ghent University and Ghent University's Special Research Fund (GOA - Concerted Research Action BOF13/GOA/032).

\section{Corresponding author:}

Evy Woumans

Department of Experimental Psychology

Ghent University

Henri Dunantlaan 2,

B9000, Gent, Belgium

Tel: 0032474548346

Email: evy.woumans@ugent.be 


\begin{abstract}
In a recent review, Mukadam, Sommerlad, and Livingston (2017) argue that bilingualism offers no protection against cognitive decline. The authors examined the results of 13 studies (five prospective, eight retrospective) in which monolinguals and bilinguals were compared for cognitive decline and onset of dementia symptoms. Analysis of four of the five prospective studies resulted in the conclusion that there was no difference between monolinguals and bilinguals, whereas seven of the eight retrospective studies actually showed bilingualism to result in a four-to-five year delay of symptom onset. The authors decided to ignore the results from the retrospective studies in favour of those from the prospective studies, reasoning that the former may be confounded by participants' cultural background and education levels. In this commentary, we argue that most of these studies actually controlled for these two variables and still found a positive effect of bilingualism. Furthermore, we argue that the meta-analysis of the prospective studies is not complete, lacking the results of two crucial reports. We conclude that the literature offers substantial evidence for a bilingual effect on the development of cognitive decline and dementia.
\end{abstract}

Key words: cognitive decline; multilingualism; dementia; prospective cohort studies; retrospective studies 
In a recent systematic review and partial meta-analysis, published in this journal, Mukadam, Sommerlad, and Livingston [1] argue that bilingualism offers no protection against cognitive decline and dementia. They conclude that "public health policy should therefore remove recommendations regarding bilingualism as a strategy to delay dementia" (p. 53). The authors examined the results of 13 studies (five prospective, eight retrospective) in which monolinguals and bilinguals were compared for cognitive state and onset of dementia symptoms. It is striking that only four of the five prospective studies and none of the retrospective studies were included in their meta-analysis. Their conclusions are based solely on the fact that this rather selective meta-analysis showed an overall unadjusted odds ratio of 0.96 for developing dementia in bilinguals versus monolinguals. Conclusions ignore seven out of eight retrospective studies that reported a bilingual delay of four to five years in dementia onset. We argue that it is questionable to include only (part of the) prospective studies in a meta-analysis, even though retrospective studies are just as informative and twice as prevalent. In addition, we also identify some ignored prospective investigations that did show a bilingual advantage in cognitive decline.

\section{Including all evidence}

In order to be valid, a systematic review and meta-analysis needs to include all relevant studies. Here, a longitudinal cohort study by Wilson and colleagues [2] reporting reduced risk of MCI in foreign language speakers was left out of the final analyses of prospective studies. The authors did not incorporate this study because, supposedly, "there was no record of whether or not participants spoke more than one language" (p. 46-47). However, the longitudinal assessment included 964 residents from the Chicago area of whom 576 received one to four years of foreign language instruction, 124 more than four years, and 264 none at all. It seems safe to state that 
the last group should be regarded as monolingual, whereas the two other should be considered bilingual, in accordance with common definitions in literature on bilingualism [3]. Across all included studies, specific criteria for bilingual status varied greatly, and it seems very ad-hoc to pick out one as a reason for exclusion, especially when only four other prospective studies were available and when that one study is inconsistent with the meta-analysis' conclusion.

Surprisingly, two (out of the five available) cohort studies that actually employed more inadequate definitions of bilingualism but did not show any bilingual effect were retained for analyses. As acknowledged by the authors themselves, Zahodne and colleagues [4] did not include any true monolinguals, as all participants were Spanish speakers, but lived in English-speaking Northern Manhattan. The same was the case for the study by Lawton and colleagues [5], who included participants with knowledge of a second language (again English) into their cohort of monolinguals.

Another of the five prospective studies [6] - again one that did reveal a cognitive advantage for bilinguals - was also not included in the meta-analysis as it measured cognitive function rather than incident dementia, even though inclusion criteria stated that cognitive scores as well as incident dementia or incident MCI were taken up in the review. The study - which was most incorrectly termed by Mukadam and colleagues as "low quality" - actually entailed a large homogeneous Scottish cohort, tested over the course of 60 years, in which participants functioned as their own baseline. Interestingly, people who became bilingual during this time period performed significantly better than predicted from their own initial cognitive abilities. The decision to cut this study from the final analysis together with the abovementioned issues make Mukadam et al.'s attempt to review the relationship 
between bilingualism and cognitive decline far from systematic and biased in favour of the null hypothesis.

\section{The question of culture}

Even though (eight) retrospective studies constitute the bulk of the available evidence, Mukadam and colleagues also dismiss them for their conclusion. They strongly argue that informant reports - such as those employed in retrospective studies - may be influenced by cultural factors. Stating that people from minority ethnic backgrounds may consult professionals at a later time point, they fail to acknowledge the retrospective research that actually took factors like immigration status into account. Of the eight studies in total, three studies $[7,8,9]$ included no immigrant samples at all in order to control for cultural background, whereas another [10] made a clear distinction between immigrant and non-immigrant bilingual samples, and compared both to the monolingual sample. Two of the three studies [7, 9] comprising of only native populations reported a four to five year delay of dementia symptoms in bilinguals.

\section{Educational accomplishment}

Another point raised by the authors is that differences in levels of education may skew the outcome of retrospective studies. However, only five of the eight studies included in the review reported such a difference between monolinguals and bilinguals; two of which $[11,12]$ actually demonstrated significantly more years of schooling for monolinguals. If higher education protects against cognitive decline [13], it would be the monolinguals who are advantaged here. The other three studies

reporting differences $[4,7,9]$ controlled for them in their analyses and found no effect of the variable, nor any interaction with bilingualism. Those studies finding later 
onset of dementia symptoms in bilinguals [7,9] also controlled for occupation, making it unlikely that socioeconomic status affected the outcome.

Although we very much appreciate the attempt made by Mukadam and colleagues to report a systematic review on the literature concerning bilingualism and cognitive decline, we feel the overview is not complete and analyses are biased towards the null hypothesis. If the entire relevant literature is considered, it becomes clear that there is considerable empirical support for a bilingual effect on dementia and cognitive decline, in different samples and contexts, controlling for a wide variety of variables. Importantly, the effects described in these studies are of a size to which no pharmacologic intervention can yet aspire. 


\section{References}

[1] Mukadam N, Sommerlad A, \& Livingston G (2017) The relationship of bilingualism compared to monolingualism to the risk of cognitive decline or dementia: A systematic review and meta-analysis. J Alzheimers Dis 58, 45-54.

[2] Wilson RS, Boyle PA, Yang J, James BD, Bennett DA (2015) Early life instruction in foreign language and music and incidence of mild cognitive impairment. Neuropsychology 29, 291-302.

[3] Grosjean F (1997) The bilingual individual. Interpreting 2, 163-187.

[4] Zahodne LB, Schofield PW, Farrell MT, Stern Y, Manly JJ (2014) Bilingualism does not alter cognitive decline or dementia risk among Spanish-speaking immigrants. Neuropsychology 28, 238-246.

[5] Lawton DM, Gasquoine PG, Weimer AA (2015) Age of dementia diagnosis in community dwelling bilingual and monolingual Hispanic Americans. Cortex 66, 141-145.

[6] Bak TH, Nissan JJ, Allerhand MM, Deary IJ (2014) Does bilingualism influence cognitive aging? Ann Neurol 75, 959-963.

[7] Alladi S, Bak TH, Duggirala V, Surampudi B, Shailaja M, Shukla AK, Chaudhuri JR, Kaul S (2013) Bilingualism delays age at onset of dementia, independent of education and immigration status. Neurology 81, 1938-1944.

[8] Clare L, Whitaker CJ, Craik FI, Bialystok E, Martyr A, Martin-Forbes PA, Bastable AJM, Pye KL, Quinn C, Thomas EM, Gathercole VC, Hindle JV (2016) Bilingualism, executive control, and age at diagnosis among people with early-stage Alzheimer's disease in Wales. J Neuropsychol 10, 163-185. 
[9] Woumans E, Santens P, Sieben A, Versijpt J, Stevens M, Duyck W (2015) Bilingualism delays clinical manifestation of Alzheimer's disease. Biling Lang Cogn 18, 568-574.

[10] Chertkow H, Whitehead V, Phillips N, Wolfson C, Atherton J, Bergman H (2010) Multilingualism (but not always bilingualism) delays the onset of Alzheimer disease: Evidence from a bilingual community. Alzheimer Dis Assoc Disord 24, 118-125.

[11] Bialystok E, Craik FI, Freedman M (2007) Bilingualism as a protection against the onset of symptoms of dementia. Neuropsychologia 45, 459-464.

[12] Craik FI, Bialystok E, Freedman M (2010) Delaying the onset of Alzheimer disease: Bilingualism as a form of cognitive reserve. Neurology 75, 1726-1729.

[13] Valenzuela MJ, Sachdev P (2006) Brain reserve and dementia: A systematic review. Psychol Med 36, 441-454. 